\title{
Histerectomía total laparoscópica en pacientes con endometriosis profunda
}

\author{
Inés Poveda G. ${ }^{1}$, Isauro Rogelio Monfort 0. $^{1}$, Nuria García C. ${ }^{1}$, Cristina Signes . $^{1}$, \\ Susana Monzó $F^{1}$, Javier Monleón $S^{1}{ }^{1}$, Antonio Abad C. ${ }^{1}$, Vicente Payá A. ${ }^{1}$ \\ ${ }^{1}$ Servicio de Ginecología, Hospital Universitario y Politécnico La Fe. Valencia, España.
}

\section{RESUMEN}

Objetivo: Analizar los resultados perioperatorios y la evolución clínica en pacientes con endometriosis profunda sometidas a histerectomía laparoscópica. Métodos: Estudio retrospectivo de 22 pacientes histerectomizadas, por endometriosis profunda entre enero de 2013 y julio de 2015 . Analizamos los resultados perioperatorios (tiempo quirúrgico, días de estancia y complicaciones) y la clínica de endometriosis (dispareunia, disquecia, disuria y dolor pélvico crónico), mediante la escala visual analógica (EVA). Resultados: El tiempo operatorio medio fue de 189 minutos y la estancia hospitalaria 4 días. La tasa de reconversión a laparotomía fue del 18\% (4 pacientes). En 6 pacientes (27\%) hubo complicaciones posoperatorias, todas menores. Tras la cirugía todas las variables mejoraron de forma significativa siendo la más beneficiada el dolor pélvico crónico (descenso medio de 7,15 en la EVA). Conclusiones: La histerectomía total es aceptable en aquellas pacientes con endometriosis profunda, con deseo genésico cumplido y sintomatología resistente a otros tratamientos.

\section{PALABRAS CLAVE: Endometriosis profunda, dolor pélvico crónico, histerectomía laparoscópica}

\section{SUMMARY}

Objective: To study the symptoms prior to surgery and 6 months after surgery in patients with deep endometriosis who underwent laparoscopic histerectomy. Methods: Retrospective study of perioperatory results (surgical time, days of stay, blood loss and complications) and endometrosis symptoms (dyspareunia, dyschezia, dysuria and chronic pelvic pain with the visual analogic scale) of 22 patients diagnosed with deep endometriosis who underwent histerectomy. Results: After the surgery all the variables improved significantly, being the chronic pelvic pain the one which improved the most (mean descent of 7.15 points in the visual analogic scale). The mean surgical time was 189 minutes, the hospital stay was 4 days. The conversion to laparotomy rate was $18 \%$ (4 patients). There were 6 minor postoperative complications $(27 \%)$. Conclusions: Total histerectomy is suitable for those patients with deep endometriosis, with fulfilled reproductive desire and symptoms that are resistant to other treatments.

KEY WORDS: Deep endometriosis, chronic pelvic pain, laparoscopic hysterectomy 


\section{INTRODUCCIÓN}

La endometriosis es un proceso inflamatorio que se caracteriza por la presencia de tejido endometrial fuera del útero, principalmente en los ovarios, aunque también puede afectar al peritoneo, el tabique rectovaginal, e incluso a órganos extrapélvicos, como el hígado o el cerebro (1). Esta patología afecta globalmente a 176 millones de mujeres (2). Afecta al 6-10\% de las mujeres en edad fértil, al $50-60 \%$ con dolor pélvico y hasta el $50 \%$ de mujeres con esterilidad $(1,3,4)$.

Hemos de sospechar esta patología ante pacientes que presenten dolor pélvico crónico, dismenorrea, dispareunia y disquecia. Estos síntomas pueden llegar a ser incapacitantes, sobre todo cuando el tejido ectópico infiltra el fondo de saco de Douglas y los ligamentos útero sacros (5).

El tratamiento de esta enfermedad debe adaptarse a la intensidad de los síntomas, la extensión y localización de las lesiones, el deseo genésico y la edad de la paciente $(1,4,6)$. Si se opta por un tratamiento conservador, puede que no sea suficiente (7). Por ello, son estas pacientes las principales candidatas a la histerectomía, sobre todo si ya han cumplido su deseo genésico $(2,4,8,9,10,11)$. Es importante la adecuada selección de estas pacientes, ya que son cirugías agresivas, no exentas de riesgos y no todas las pacientes se benefician de esta técnica.

El objetivo de este estudio es analizar la evolución clínica de estas pacientes a los 6 meses tras la cirugía y los resultados perioperatorios de las histerectomías realizadas con el diagnóstico de endometriosis profunda.

\section{PACIENTES Y MÉTODOS}

Estudio retrospectivo de práctica clínica de las pacientes sometidas a histerectomía total laparoscópica por endometriosis profunda, entre enero de 2013 y julio de 2015, en el Hospital Universitario y Politécnico La Fe.

Las variables analizadas fueron las relacionadas con la clínica de la endometriosis (dismenorrea, dispareunia, disquecia, disuria y dolor pélvico crónico). Las variables perioperatorias estudiadas fueron la edad, paridad, tiempo quirúrgico, estancia hospitalaria, complicaciones intra y postquirúrgicas.

Un total de 22 pacientes fueron seleccionadas en este período. Todas las cirugías fueron realizadas por 4 cirujanos con experiencia en cirugía de endometriosis.

El estudio de extensión preoperatorio realizado en todos los casos consistió en exploración física mediante tacto vaginal, ecografía transvaginal y transrectal si se sospechaba afectación intestinal. Se hizo resonancia magnética en todos los casos.
Se realizó enema opaco en dos casos debido a la alta sospecha de afectación de la luz intestinal informada en la resonancia.

Todas las pacientes que aceptaron ser intervenidas fueron informadas antes de la intervención, de la técnica quirúrgica, sus riesgos, complicaciones y posibles alternativas, reflejándose todo esto en el consentimiento informado, el cual se obtuvo y fue firmado previo a la cirugía por todas las pacientes.

Las variables perioperatorias se obtuvieron de la historia clínica informatizada. El tiempo quirúrgico se calculó desde la primera incisión hasta el cierre de la cúpula vaginal. La pérdida de sangre durante la operación se halló restando al valor preoperatorio de la hemoglobina el valor de la misma 24 horas después de la intervención, este control analítico fue realizado en todas las pacientes. El tiempo de ingreso postoperatorio se calculó desde el día de la cirugía hasta el del alta hospitalaria.

Los síntomas relacionados con la endometriosis (dismenorrea, disquecia, disuria, dispareunia, dolor pélvico crónico) se expresaron según la escala visual analógica (EVA), se compararon los valores previos a la cirugía con los referidos por la paciente 6 meses después de la cirugía. En el caso de la dismenorrea solo se disponen de los datos preoperatorios. Las variables cuantitativas se expresan en media y desviación estándar, y las proporciones en porcentajes. El análisis estadístico se realizó mediante el test de t de Student para datos apareados. Se considera un valor $p<0,05$ como estadísticamente significativo.

Técnica quirúrgica. La intervención se realizó con anestesia general, profilaxis antibiótica y anticoagulación profiláctica con heparina de bajo peso molecular. Las pacientes se colocaron en decúbito dorsal con los miembros inferiores en leve abducción. Se practicó un tacto bimanual bajo anestesia para valorar el tamaño y la movilidad del útero, se dejó una sonda vesical permanente y se colocó el movilizador uterino de Clermont-Ferrand (Karl Storz Endoskope, Tuttlingen, Alemania). El neumoperitoneo se realizó con aguja de Veress en punto de Palmer o infraumbilical. Se introdujeron 4 trocares, uno de $10 \mathrm{~mm}$ para la óptica, que se coloca infraumbilicalmente. Dos trocares laterales de 5 $\mathrm{mm}$ en ambas fosas ilíacas y otro de $5 \mathrm{~mm}$ en línea media del área suprapúbica. La pinza de corriente bipolar utilizada fue la Biclamp ${ }^{\circledR}$ (ERBE Elektromedizin GMBH, Tuebingen, Alemania).

La técnica quirúrgica utilizada en todas las histerectomías, con variaciones dependiendo de la dificultad del procedimiento, es la descrita en la literatura con pinza bipolar y tijera fría (12), extirpando todas las lesiones endometriósicas que afectaban al peritoneo, ovarios, fondo de saco de Douglas, tabique recto-vaginal, parametrios, recto-sigma y 
vejiga. Para ello, antes iniciar la histerectomía se liberaron los ovarios del ligamento ancho, los uréteres y el recto de la pared posterior del útero y vagina exponiendo el espacio rectovaginal. Se realizó cistectomía parcial en un caso. En los 2 casos que se realizó resección intestinal, esta fue realizada por un cirujano.

La extracción del útero se hizo por vagina tras colpotomía circular con corriente monopolar de corte (70-80 Watt). El cierre de la vagina se hizo con puntos sueltos.

\section{RESULTADOS}

En la Tabla I se muestran las características de las pacientes. La edad media era de 42,3 años, siendo la mayoría $>40$ años (82\%). Destaca que 7 (32\%), aun siendo nulíparas fueron histerectomizadas y $16(73 \%)$ tenían una laparotomía abdominal previa, de las cuales 13 casos había sido por endometriosis. En todas ellas existía afectación del compartimento posterior, en 11 casos el lateral y en una paciente el anterior. Los ovarios estaban afectados en todos los casos.

En todas las pacientes se realizó histerectomía más anexectomía con adhesiolisis y extirpación de implantes endometriósicos en forma de placas o nódulos. En 19 pacientes la anexectomía fue bilateral y en 3 pacientes unilateral ya que uno de los anexos se había extirpado en cirugía previa por endometriosis. En una paciente con afectación del compartimento anterior se realizó cistectomía parcial para resecar un nódulo vesical.

En la Tabla II figuran los resultados y complicaciones perioperatorias. El tiempo quirúrgico medio fue superior a 3 horas y la estancia hospitalaria media de 4 días. Hubo sólo una complicación intraoperatoria, una perforación intestinal al liberar el sigma de los anexos y que se solucionó por laparotomía. Hubo otras 3 reconversiones a laparotomía, todas ellas por imposibilidad de liberar las adherencias por vía laparoscópica. En 2 pacientes se realizó resección intestinal y sutura termino terminal por parte de un cirujano general experimentado. En el posoperatorio hubo 6 complicaciones posoperatorias todas menores (27\%): 2 abscesos de cúpula vaginal, 2 íleos paralíticos y dos fiebres posquirúrgicas sin foco conocido. La hemoglobina cayó de media 2,5 g/dl precisando tratamiento con hierro 11 pacientes $(50 \%)$. No se realizó transfusión de concentrados de hematíes a ninguna paciente.

Finalmente, la Tabla III muestra la evolución de la clínica previa a la cirugía y 6 meses tras la cirugía. En cuanto al estudio del dolor postquirúrgico, sólo se pudo contactar con 21 de las 22 pacientes, por tanto el análisis se limitará a estas pacientes. Se estudia la evolución de 4 variables clínicas: la dispareunia, la disquecia, la disuria y el dolor pélvico crónico.

La dismenorrea prequirúrgica estaba presente en todas las pacientes con un EVA medio de 9,17 puntos.

La dispareunia estaba presente en 17 de las 21 pacientes $(80 \%)$ antes de la intervención, con un EVA medio de 8,35 puntos. Tras la cirugía mejoró en 13 pacientes $(76 \%)$ y persistió en $3(18 \%)$. Una de las pacientes no ha mantenido relaciones tras la cirugía. La mejoría media fue de 5,61 puntos en la escala EVA.

Tabla I

\section{CARACTERÍSTICAS DE LAS PACIENTES}

\section{Edad (años)}

Pacientes $<40$ años

IMC $\left(\mathrm{kg} / \mathrm{cm}^{2}\right)$

Paridad

Menopausia

Laparotomía previa
$42,32 \pm 4,57$ (Rango: 35-50)

$4(18 \%)$

$24,99 \pm 3,76$ (Rango: 19,72-31,64)

Nulíparas: $31,81 \%$ (n: 7)

Parto o cesárea previa: $68,18 \%$ (n: 15)

$22(100 \%)$

No: $27,27 \%$ (n: 6)

Sí: $72,72 \%$ (n: 16)

- CST: 7

- LPT por endometriosis: 13

- Otras LPT: 3 


\section{Tabla II RESULTADOS PERIOPERATORIOS}

Indicación cirugía
Compartimento afectado
Tipo de cirugía
Tiempo quirúrgico (min)
Estancia hospitalaria (días)
Complicaciones intraoperatorias
Reconversión a laparotomía
Complicaciones postquirúrgicas

Caída Hb (g/dl)
Endometriosis profunda: $100 \%$

Posterior: $22(100 \%)$

Lateral: $11(50 \%)$

Anterior: $1(4,6 \%)$

HST + DA + AD: $100 \%(22)$

HST + AU: 13,6\% (3)

189,27 $\pm 71,12$ (Rango: 90-315)

4,09 \pm 1,66 (Rango: $2-8$ )

Perforación intestinal: 1

$18,18 \%(4)$

Absceso cúpula: 9,09\% (2)

Íleo: 9,09\% (2)

Fiebre post cirugía: 9,09\% (2)

$2,53 \pm 1,25$ (Rango: 0,4-4,7)

HST: histerectomía. DA: doble anexectomía. AU: anexectomía única. AD: adhesiolisis. Hb: hemoglobina.

\section{Tabla III}

EVOLUCIÓN CLÍNICA 6 MESES TRAS LA CIRUGÍA

\begin{tabular}{|c|c|c|c|c|c|c|}
\hline Variables & $\begin{array}{c}\text { Presencia } \\
\text { n (\%) }\end{array}$ & $\begin{array}{c}\text { EVA } \\
\text { preoperat. }\end{array}$ & $\begin{array}{c}\text { EVA } \\
\text { postoperat. }\end{array}$ & $\begin{array}{l}\text { Diferencia } \\
\text { EVA }\end{array}$ & $\begin{array}{l}\text { Evolución } \\
\text { M (mejor) } \\
\text { P (peor) } \\
\text { I (igual) }\end{array}$ & Valor $\mathrm{P}$ \\
\hline Dismenorrea & $22(100)$ & $9,17 \pm 1,19$ & - & - & - & - \\
\hline Dispareunia & $17(80)$ & $8,35 \pm 1,80$ & $3,69 \pm 3,66$ & $4,56 \pm 3,01$ & $\begin{array}{l}\text { M: } 13 / 17(76,5 \%) \\
P: 0 \\
\text { I: } 3(17,6 \%) \\
1 \text { caso sin RRSS tras Cx }\end{array}$ & 0,0001 \\
\hline Disquecia & $15(71,4)$ & $7,47 \pm 2,59$ & $2,13 \pm 2,83$ & $5,33 \pm 3,09$ & $\begin{array}{l}\text { M: } 14 / 15(99,3 \%) \\
\text { P: } 0 \\
\text { I: } 1(17,6 \%) \\
1 \text { caso de nueva apari- } \\
\text { ción tras Cx (EVA 5) }\end{array}$ & 0,0001 \\
\hline Disuria & $6(28,6)$ & $5,83 \pm 1,60$ & $0,33 \pm 0,82$ & $5,50 \pm 1,52$ & $\begin{array}{l}\text { M: } 6 / 6(100 \%) \\
\text { P: } 0 \\
\text { I: } 0 \\
4 \text { casos de nueva } \\
\text { aparición tras Cx (EVA } \\
3 \pm 2,71)\end{array}$ & 0,0003 \\
\hline $\begin{array}{l}\text { Dolor pélvico } \\
\text { crónico }\end{array}$ & $20(95,2)$ & $8,15 \pm 2,28$ & $1 \pm 1,65$ & $7,15 \pm 2,48$ & $\begin{array}{l}\text { M: } 20 / 20(100 \%) \\
P: 0 \\
\text { I: } 0\end{array}$ & 0,0001 \\
\hline
\end{tabular}

EVA: escala visual análogica. RRSS: relaciones sexuales. Cx: cirugía. 
La disquecia estaba presente en 15 de las 21 $(71,4 \%)$ pacientes, con un EVA medio de 7,46 puntos. Tras la cirugía mejoró en 14 pacientes (media de 5,71 puntos), se mantuvo en 1 y apareció en otra paciente, que refiere problemas de estreñimiento crónico tras la cirugía.

La disuria estaba presente en $6(28,6 \%)$ pacientes, con una media de 5,83 puntos. Mejoró en todas ellas, la media de la mejoría fue 5,5 puntos. En 4 pacientes apareció disuria tras la cirugía.

El dolor pélvico crónico estaba presente en 20 $(95,2 \%)$ pacientes antes de la intervención, siendo el EVA medio de 8,15 puntos. Mejoró en todas ellas, de media de 7,15 puntos. Una paciente no presentaba este dolor incluso previo a la cirugía.

\section{DISCUSIÓN}

La endometriosis es un proceso crónico que puede resultar incapacitante para determinadas pacientes. Cuando fracasan las opciones conservadoras de tratamiento, la única opción para estas pacientes es la cirugía con extirpación de útero y anexos.

Nuestros resultados ponen de manifiesto que la histerectomía con doble anexectomía está justificada en aquellas pacientes con clínica de dolor pélvico muy intenso que les incapacita para llevar a cabo su actividad habitual y que tienen su deseo genésico cumplido $(2,4,8,9,10,11)$. Llama la atención que en 7 pacientes, a pesar de ser nulíparas, al valorar todas las opciones, se decidió el tratamiento radical debido a la severidad de los síntomas.

En nuestro centro realizamos histerectomía con anexectomía bilateral, pues como indican otros trabajos previos, pensamos que el tratamiento radical de la endometriosis mejora la sintomatología y disminuye significativamente la tasa de recidivas y reintervenciones $(7,9,10,13)$. Posteriormente, al ser tratadas con estrógeno-progestativos o tibolona el riesgo de recurrencia es marginal $(14,15)$. Actualmente, 12 de estas pacientes están siendo tratadas con tratamiento hormonal sustitutivo.

Otro aspecto de máxima importancia es que este tipo de cirugías deben ser realizadas por cirujanos con experiencia y dentro de un equipo multidisciplinar, puesto que suelen ser largas (189 minutos de media) y no están exentas de riesgo. En nuestra serie, a pesar de que hubo 4 conversiones a laparotomía, la vía inicial de abordaje fue la laparoscópica, la cual ha demostrado menor tasa de complicaciones que la laparotómica $(4,16,17)$.

Como ya se ha mencionado, tuvimos una complicación intraoperatoria que se solucionó durante la cirugía y 6 complicaciones posoperatorias (27\%), todas ellas menores. No tuvimos en nuestra serie ninguna lesión de uréteres, atribuible a la técnica de la histerectomía, que consiste en una exposición de los mismos antes de iniciar la histerectomía.

Centrándonos en el objetivo principal del estudio, al analizar la evolución de estas pacientes a los 6 meses tras la cirugía, los resultados son favorables, reflejados en la mejoría de todas las variables estudiadas. Otros autores abordan esta cuestión. Así, Lamvu (18) refiere que un 14\% de las mujeres operadas tendrán un resultado postoperatorio peor que el esperado, lo que también ocurre en nuestra serie no mejorando la dispareunia en 3 casos (17,6\%) y la disquecia en $1(6,6 \%)$. Para Rizk y cols (2), la persistencia de la enfermedad tras la intervención quirúrgica es la causa principal de que esta cirugía no sea definitiva, y también, como indica Lamvu (18), todas las pacientes con dolor pélvico crónico que son histerectomizadas por endometriosis deberían ser evaluadas antes de la intervención para excluir otras causas de dolor pélvico no ginecológico.

\section{CONCLUSIÓN}

Podemos afirmar que la histerectomía con anexectomía es una técnica apropiada para el tratamiento de la endometriosis profunda en casos seleccionados, tras fracaso de medidas conservadoras. Pese a ser llevada a cabo por cirujanos experimentados, no está exenta de riesgos. Es importante tener en cuenta que no todas las pacientes presentan los beneficios esperados de esta técnica. Son necesarios más estudios con mayor cantidad de pacientes, más seguimiento a largo plazo para poder evolucionar en el tratamiento y el seguimiento de estas pacientes.

\section{REFERENCIAS}

1. Giudice LC. Endometriosis. N Engl J Med 2010;362:2389-98.

2. Rizk B, Fischer AS, Lotfy HA, Turki R, Zahed HA, Malik $R$, et al. Recurrence of endometriosis after hysterectomy. Facts View Vis ObGyn 2014;6(4):219-27.

3. Kennedy S, Bergquist A, Chapron C, D'Hooghe T, Dunselman G, Greb Hummelshoj L, et al. ESHRE guideline for the diagnosis and treatment of endometriosis. Hum Reprod 2005;20:2698-704.

4. Chalermchockchareonkit A, Tekasakul P, Chaisilwattana $P$, Sirimai K, Wahab N. Laparoscopic hysterectomy versus abdominal hysterectomy for severe pelvic endometriosis. J Gynaecol Obstet 2012;116:109-11.

5. Vercellini $P$, Carmignani L, Rubino $T$, Barbara $G, A b-$ biati A, Fedele L. Surgery for deep endometriosis: a pathogenesis-oriented approach. Gynecol Obstet Invest 2009;68:88-103.

6. Donnez J, Pirard C, Smets M, Jadoul P, Squifflet J. Surgical management of endometriosis. Best Pract Res Clin Obstet Gynaecol 2004;18:329-48.

7. Abbott JA, Hawe J, Clayton RD, Garry R. The effects and effectiveness of laparoscopic excision of endometriosis: a prospective study with $2-5$ year follow-up. Hum Reprod 2003;18:1922-7. 
8. Weir E, Mustard C, Cohen M, Kung R. Endometriosis: what is the risk of hospital admission, readmission, and major surgical intervention? J Minim Invasive Gynecol 2005;12:486-93.

9. Shakiba K, Bena JF, McGill KM, Minger J, Falcone T. Surgical treatment of endometriosis: a 7-year followup on the requirement for further surgery. Obstet Gynecol 2008; 111:1285-92.

10. Namnoum AB, Hickman TN, Goodman SB, Gehlbach DL, Rock JA. Incidence of after hysterectomy for endometriosis symptom recurrence. Fertil Steril 1995;64:898-902.

11. Hansen K A, Chalpe A, Eyster KM. Management of endometriosis-associated pain. Clin Obstet Gynecol 2010;53:439-48.

12. Wattiez A, Thoma V, Nassif J. Laparoscopic total hysterectomy for benign conditions: standard technique. Epublication: WeBSurg.com, 2008;8(3). Disponible en: http://www.websurg.com/ref/doi-ot02en326.htm.

13. Fedele L, Bianchi S, Zanconato G, Berlanda N, Borruto $F$, Frontino $G$. Tailoring radicality in demolitive surgery for deeply in infiltrating endometriosis. Am J Obstet Gynecol 2005;193(1):114-7
14. Matorras R, Elorriaga MA, Pijoan Jl, Ramón O, Rodríguez-Escudero FJ. Recurrence of endometriosis in women with bilateral adnexectomy (with or without total hysterectomy) who received hormone replacement therapy. Fertil Steril 2002;77(2):303-8.

15. Vercellini P, Barbara G, Abbiati A, Somigliana E, Viganò $P$, Fedele $L$. Repetitive surgery for recurrent symptomatic endometriosis: what to do? Eur J Obstet Gynecol Reprod Biol 2009;146(1):15-21.

16. Wolthius AM, Meuleman C, Tomassetti C, D'Hooghe T, van Overstraeten A, D'Hoore A. Bowel endometriosis: Colorectal surgeon's perspective in a multidisciplinary surgical team. World J Gastroenterol 2014;20(42):15616-23.

17. Daraï E, Dubernard G, Coutant C, Frey C, Rouzier $\mathrm{R}$, Ballester M. Randomized trial of laparoscopically assisted versus open colorectal resection for endometriosis: morbidity, symptoms, quality of life, and fertility. Ann Surg 2010;251(6):1018-23.

18. Lamvu G. Role of hysterectomy in the treatment of chronic pelvic pain. Obstet Gynecol 2011;117(5):11758. 